\title{
Exogenous ochronosis in a Chinese patient: use of dermoscopy aids early diagnosis and selection of biopsy site
}

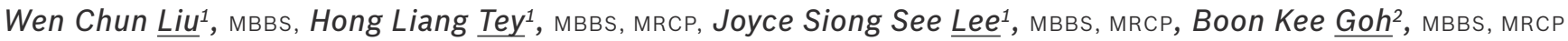

\begin{abstract}
The diagnosis of exogenous ochronosis is often challenging and requires a high index of suspicion. Herein, we report a case of exogenous ochronosis in a Chinese patient. The condition was caused by the use of bleaching agents, including creams containing hydroquinone. We demonstrate the use of dermoscopy as an invaluable tool for the early recognition of the condition, as well as in the selection of an appropriate site for a skin biopsy.
\end{abstract}

Keywords: dermoscopy, hydroquinone, melasma, ochronosis

\section{INTRODUCTION}

The term 'ochronosis' was first coined by Virchow in 1866 to refer to brownish-yellow accumulations of pigment found in the connective tissue of patients with endogenous ochronosis. ${ }^{(1)}$ Endogenous ochronosis, also known as alkaptonuria, is a rare $(1: 250,000)$ autosomal recessive disease caused by the congenital absence of homogentisic acid oxidase, leading to the accumulation of homogentisic acid within various tissues of the body. ${ }^{(2)}$ Cutaneous features of endogenous ochronosis include blue-black hyperpigmentation on the pinnae, nasal tip, cheeks, sclerae and axilla. Systemic deposition of homogentisic acid results in ochronotic arthropathy (mainly involving large joints and the vertebral column), deafness, genitourinary obstruction and cardiovascular complications (e.g. aortic valve calcification and stenosis). Diagnosis is usually made clinically or with urine tests using sodium hydroxide.

Exogenous ochronosis was first described by Pick in 1906. ${ }^{(3)}$ This condition is often reported in the black population of South Africa as a result of the use of bleaching agents, particularly hydroquinone. ${ }^{(4)}$ Other agents implicated include antimalarials, and products containing resorcinol, phenol, mercury or picric acid. ${ }^{(5)}$ Classical features of exogenous ochronosis are asymptomatic blue-black macules with textural coarsening and sooty tipped papulations on the malar area, temples, inferior cheeks and neck. In 1979, Dogliotti et $\mathrm{al}^{(6)}$ classified exogenous ochronosis into three clinical stages: (a) erythema and mild hyperpigmentation; (b) hyperpigmentation, pigmented colloid milium (i.e. caviar-like lesions) and scanty atrophy; and (c) papulonodular lesions.

Recently, $\operatorname{Tan}^{(7)}$ described a case series of exogenous ochronosis in the Chinese population. In that study, Tan highlighted that the actual prevalence of the condition in the Chinese population was likely to be higher than that reported,

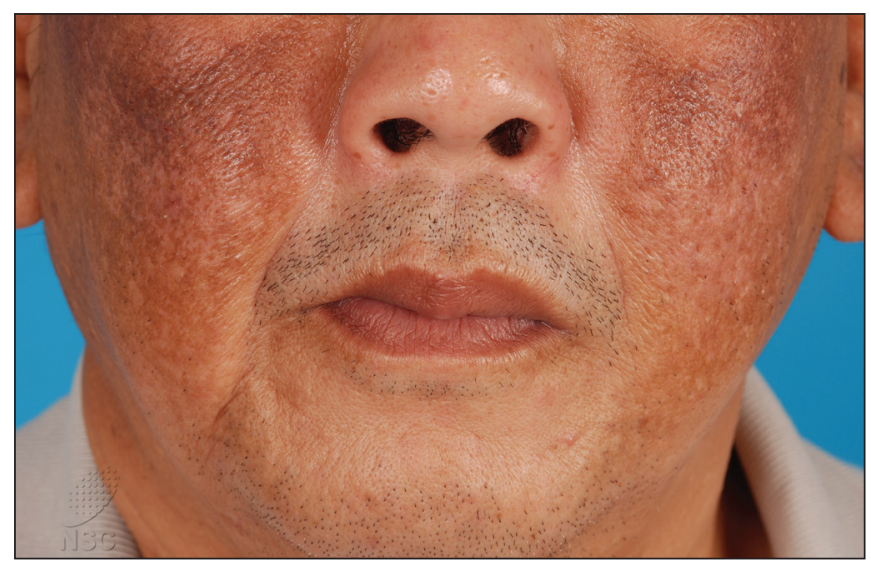

Fig. 1 Photograph shows the features of ochronosis observed in our patient: velvety, greyish-brown patches with coarse papulations; and scattered confetti-like hypopigmented macules secondary to chemical leucoderma.

as cases are often misdiagnosed as dermal or refractory melasma. Herein, we report a case of exogenous ochronosis in a 50-year-old Chinese male after prolonged treatment with skin bleaching agents. We also highlight the use of dermoscopy in aiding diagnosis and the selection of a site for skin biopsy.

\section{CASE REPORT}

A 50-year-old Chinese man with skin phototype IV presented to our institution with a chronic history of bilateral hyperpigmentation over the malar area. Over the years, the patient had tried various over-the-counter, homeopathic and prescribed bleaching agents, including creams containing hydroquinone at various concentrations. While there was initial improvement after treatment with those various agents, the hyperpigmentation gradually became darker in the last one year despite sun protection. He had a medical history of hypertension, hypercholesterolaemia, type 2 diabetes mellitus and ischaemic

${ }^{1}$ National Skin Centre, ${ }^{2}$ Skin Physicians, Mount Elizabeth Medical Centre, Singapore

Correspondence: Dr Wen Chun Liu, Senior Resident, National Skin Centre, 1 Mandalay Road, Singapore 308205. coniliu@nsc.gov.sg 


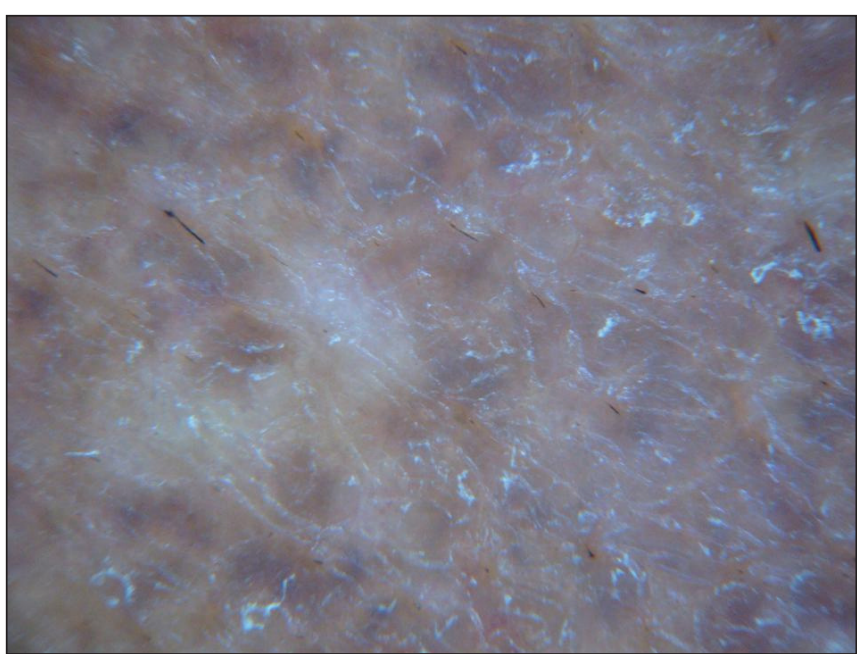

Fig. 2 Dermoscopic photograph shows scattered blue-grey dots and globules that have a caviar-like appearance, obliteration of follicular openings, and scattered structureless areas.

heart disease (a coronary bypass surgery was done in May 2009). His oral medications included atenolol, lovastatin, metformin, glipizide and aspirin. He had no skeletal, genitourinary or cardiac symptoms at the time of medical review.

Examination revealed bilateral symmetrical, confluent brown patches over the cheeks and forehead, as well as superimposed, velvety, greyish-brown patches with coarse papulations over the maxillary area. There were also scattered confetti-like hypopigmented macules present within the borders of the lesion (Fig. 1). Dermoscopic examination of the velvety areas revealed scattered blue-grey dots and globules that had a caviar-like appearance, obliteration of follicular openings, and scattered structureless areas (Fig. 2). Dermoscopy of the adjacent brown areas showed an accentuation of the normal pseudo-rete of the face.

Biopsy of the lesional skin revealed a pigmented epidermis. In the upper dermis, swollen, basophilic collagen fibres were found together with curvilinear, ochre-coloured fragmented collagen bundles. Solar elastosis and dilated vessels were also present (Fig. 3). A diagnosis of Dogliotti stage 2 exogenous ochronosis was made, and the patient was advised to stop the use of all bleaching creams. He was started on treatment with topical tretinoin and niacinamide. He was also started on a therapeutic trial of Q-switched neodymium-doped yttrium aluminium garnet (Nd-Yag) 1064-nm laser therapy; however, no improvement was seen after a total of six sessions (a fluence ranging from $6-9 \mathrm{~J} / \mathrm{cm}^{2}$ was used per session).

\section{DISCUSSION}

Melasma is a common condition in Asians, and treatment commonly involves the use of bleaching creams, including creams containing hydroquinone. Exogenous ochronosis, on the other hand, is less common and may be mistaken as melasma. Such a misdiagnosis may lead to unwanted results as exogenous ochronosis is aggravated by the use of bleaching agents

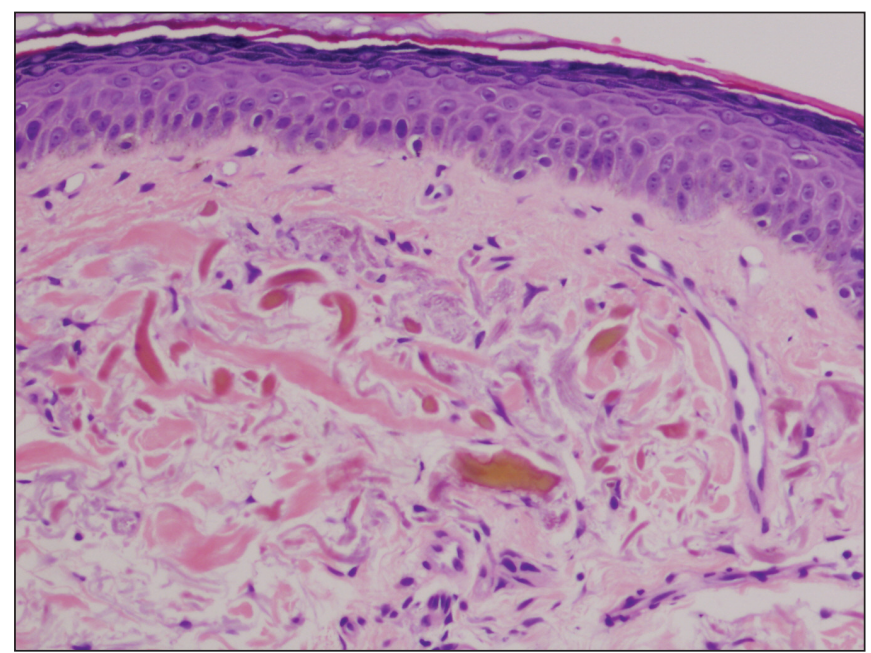

Fig. 3 Photomicrograph of the skin biopsy specimen shows early ochronotic changes of swollen, basophilic collagen fibres, and established changes of variably fragmented collagen bundles with ochre pigmentation (banana-shaped fibres) (Haematoxylin \& eosin, × 200).

containing hydroquinone. However, the diagnosis of exogenous ochronosis remains a challenge and it requires a high index of suspicion. The following features should alert the physician to a possible diagnosis of exogenous ochronosis: (a) paradoxical darkening; (b) greyish-blue hyperpigmentation; (c) velvety structures or papulations in patients on long-term bleaching agents.

Although the use of dermoscopy for the diagnosis of exogenous ochronosis is not widely practised, it should be encouraged. Charlín et $\mathrm{al}^{(8)}$ were the first to describe the dermoscopic findings of ochronosis. It was described as "bluish-gray amorphous areas obliterating the follicular structures". In contrast, melasma appears as accentuations of the normal pseudo-rete of the face. Other descriptions of exogenous ochronosis include "brown-gray globular, annular and arciform structures", ${ }^{(9)}$ and in the recent case series by Tan et al, ${ }^{(10)}$ "speckling" in ethnically Chinese patients. The dermoscopic feature in our patient with Dogliotti stage 2 exogenous ochronosis was caviar-like papules (i.e. distinctive blue-grey dots and globules obliterating follicular openings). Confetti-like leucoderma, which showed up as structureless areas on the skin, was also observed in our patient. Guttate leucoderma has also been reported in other Chinese patients with exogenous ochronosis. ${ }^{(7,10)}$ We believe this to be a result of chemical leucoderma, a side effect from other coexisting bleaching agents such as phenolic or mercurial products in unapproved, homeopathic preparations. As melasma is often a concomitant finding in ochronosis, the use of dermoscopy is a useful and practical tool to distinguish the two conditions, aiding in early diagnosis and enabling the clinician to choose an appropriate site for skin biopsy.

The pathoaetiology of exogenous ochronosis remains an enigma. Histologically, ochronosis lesions are characterised by pigment incontinence; solar elastosis; brownish-yellow (i.e. ochre), banana-shaped fibres in the papillary dermis; and eventual 
degeneration of the collagen. ${ }^{(8,11)}$ Sarcoid-like granulomas with multinucleated giant cells engulfing ochronotic particles and transfollicular elimination of ochronotic fibres have also been described. ${ }^{(12)}$ The mechanism by which these ochre-coloured particles form with hydroquinone use is subject to debate. Hydroquinone interferes with skin pigmentation through the alteration of melanosome formation and melanisation, its interaction with the copper atoms at the active site of tyrosinase, and the inhibition of deoxyribonucleic acid and ribonucleic acid synthesis. Penneys ${ }^{(13)}$ proposed that topically applied hydroquinone inhibits homogentisic acid oxidase in the skin, resulting in local accumulation of homogentisic acid, which then polymerises to form ochronotic pigment particles. These pigment particles may be elastic or collagen fibres, ${ }^{(13)}$ and it appears that melanocytes play an instrumental role in the formation of these particles. Hull and Procter ${ }^{(5)}$ reported the case of a black woman with both vitiligo and exogenous ochronosis. Despite severe ochronotic changes over the woman's forehead and malar area, only scanty pigmented papules without textural changes were observed in the areas of vitiligo. Histological findings revealed that in the areas of vitiligo where melanocytes were absent, the dermis was almost entirely free of ochronotic fibres. ${ }^{(5)}$ This finding supports the hypothesis that active melanocytes are essential for the metabolism of hydroquinone in the development of ochronosis. In addition, hydroquinone-induced ochronosis is typically heralded by an initial improvement with the use of hydroquinone, followed by gradual worsening with continuous application of hydroquinone. The overactive melanocytes in ochronosis appear to develop after the melanocytes have overcome the damaging influence of hydroquinone. Findlay et $\mathrm{al}^{(14)}$ suggested that it was only after the selective uptake of hydroquinone by melanocytes had ceased that hydroquinone (with its chemicals and metabolic derivatives) would be able to pass into the dermis and produce ochronotic pigment.

The initial belief was that only high concentrations of hydroquinone (i.e. $3.5 \%$ to $7 \%$ ) could cause ochronosis. ${ }^{(14,15)}$ However, Hoshaw et $\mathrm{al}^{(16)}$ in 1985 questioned that theory as their patients developed exogenous ochronosis with the use of over-thecounter hydroquinone creams. Over the years, more cases of exogenous ochronosis arising after the use of $2 \%$ hydroquinone preparations were reported. ${ }^{(15,16)}$ It was then purported that it was not the high concentration of hydroquinone that caused the condition, but rather the extended use of hydroquinone.
Exogenous ochronosis is notoriously difficult to treat. Various treatments (e.g. the use of tretinoin gel, cryotherapy and/or trichloroacetic acid) have been attempted without much success. Diven et $\mathrm{al}^{(17)}$ reported good improvement with the combined use of dermabrasion and carbon dioxide laser therapy. Although Q-switched lasers (ruby, alexandrite or Nd-Yag 1064-nm) were among the few treatments with promising results reported, ${ }^{(7,18)}$ our patient did not show any improvement after a trial of Q-switched Nd-Yag laser therapy. Further research is warranted in the search for a consistent treatment for exogenous ochronosis.

\section{REFERENCES}

1. Virchow R. Eii Fall von allegemeiner ochronose der knorpel and knorpelahnlichen theile. Virchows Arch (Pathol Anat) 1866; 37:212-9. German.

2. Srsen S. Alkaptouria. Johns Hopkins Med J 1979; 145:217-26.

3. Pick L. Uber die Ochronose. Klin Wochenschr 1906; 43:478-80. German.

4. Levin $\mathrm{CY}$, Maibach $\mathrm{H}$. Exogenous ochronosis. An update on clinical features, causative agents and treatment options. Am J Clin Dermatol. 2001; 2:213-7.

5. Hull PR, Proctor PR. The melanocyte: an essential link in hydroquinoneinduced ochronosis. J Am Acad Dermatol 1990; 22:529-31.

6. Dogliotti M, Leibowitz M. Granulomatous ochronosis - a cosmeticinduced skin disorder in Blacks. S Afr Med J 1979; 56:757-60.

7. Tan SK. Exogenous ochronosis in ethnic Chinese Asians: a clinicopathological study, diagnosis and treatment. J Eur Acad Dermatol Venereol 2011; 25:842-50.

8. Charlín R, Barcaui CB, Kac BK, et al. Hydroquinone-induced exogenous ochronosis: a report of four cases and usefulness of dermoscopy. Int J Dermatol 2008; 47:19-23.

9. Mishra SN, Dhurat RS, Deshpande DJ, Nayak CS. Diagnostic utility of dermatoscopy in hydroquinone-induced exogenous ochronosis. Int J Dermatol 2013; 52:413-7.

10. Tan SK, Sim CS, Goh CL. Hydroquinone-induced exogenous ochronosis in Chinese--two case reports and a review. Int J Dermatol 2008; 47:639-40.

11. Merola JF, Meehan S, Walters RF, Brown L. Exogenous ochronosis. Dermatol Online J 2008; 14:6.

12. Kramer KE, Lopez A, Stefanato CM, Phillips TJ. Exogenous ochronosis. J Am Acad Dermatol 2000; 42:869-71.

13. Penneys NS. Ochronosislike pigmentation from hydroquinone bleaching creams. Arch Dermatol 1985; 121:1239-40.

14. Findlay GH, Morrison JG, Simson IW. Exogenous ochronosis and pigmented colloid milium from hydroquinone bleaching creams. Br J Dermatol 1975; 93:613-22.

15. Lawrence N, Bligard CA, Reed R, Perret WJ. Exogenous ochronosis. J Am Acad Dermatol 1988; 18:1207-11.

16. Hoshaw RA, Zimmerman KG, Menter A. Ochronosislike pigmentation from hydroquinone bleaching creams in American blacks. Arch Dermatol 1985; 121:105-8

17. Diven DG, Smith EB, Pupo RA, Lee M. Hydroquinone-induced localized exogenous ochronosis treated with dermabrasion and $\mathrm{CO}_{2}$ laser. J Dermatol Surg Oncol 1990; 16:1018-22.

18. Bellew SG, Alster TS. Treatment of exogenous ochronosis with a Q-switched alexandrite (755 nm) laser. Dermatol Surg 2004; 30:555-8. 